\title{
The mediation effect of Perceived Social Support and Perceived Stress on the relationship between Emotional Intelligence and Psychological Wellbeing in Male Athletes
}

\author{
by \\ Romualdas Malinauskas ${ }^{1}$, Vilija Malinauskiene ${ }^{1}$
}

\begin{abstract}
This study aimed to examine the relationship between emotional intelligence (EI) and psychological wellbeing in a three-month follow-up study of male athletes. In addition, we examined the mediating role of perceived social support and perceived stress on the relationship between EI and psychological wellbeing. The sample included 398 male athletes who completed measures of emotional intelligence (Schutte Self-Report Inventory), psychological wellbeing (Ryff Psychological Wellbeing Scale; SSRI), perceived social support (Multidimensional Scale of Perceived Social Support), and perceived stress (Perceived Stress Scale-10). Results from structural equation modelling procedures identified that perceived social support and perceived stress partially mediated the association between EI and psychological wellbeing. The sequential mediation effects of perceived social support-perceived stress on the relations between EI and wellbeing were confirmed. Finally, limitations and recommendations for future research were considered.
\end{abstract}

Key words: emotional intelligence, psychological wellbeing, perceived social support, perceived stress, athletes.

\section{Introduction}

Researchers have investigated the relationship between emotional intelligence (EI) and psychological wellbeing in a number of ways and from different disciplinary perspectives, examining various associations with other variables. The researchers within the field of sport and exercise psychology have focused on potential mediating variables which can account for this relationship, for instance, perceived social support (Roohafza et al., 2016; Smith et al., 1995) and perceived stress (Laborde et al., 2016; Raedeke and Smith, 2004; Roohafza et al., 2016).

EI and psychological wellbeing

Wellbeing, in this case psychological wellbeing, represents the state of individuals whose lives are going well and is one of the most examined variables associated with EI. This study employs the Ryff's (1989) concept of psychological wellbeing as optimal psychological functioning and experience and defines EI as the ability to perceive, control, and evaluate emotions (Zeidner et al., 2012). The trait EI approach has been used because the assessment of trait EI is relatively simple (Petrides et al., 2007) and there already exist some widely used tools for its measurement (e.g., Schutte et al., 1998).

A number of studies have confirmed the relationship between EI and wellbeing in the sport context (Laborde, 2014; Petrides et al., 2007). Studies outside athletic samples have reported that EI can predict wellbeing (Gallagher and Vella-Brodrick, 2008), perceived stress (Aghdasi et al., 2011; Ciarrochi et al., 2002; Slaski and Cartwright, 2003) or social support (Gallagher and Vella-Brodrick, 2008; Montes-Berges and Augusto, 2007).

EI, perceived social support, perceived stress and psychological wellbeing

Although the scientific literature is clear that EI is associated with wellbeing and psychological wellbeing, far less is known about the processes underlying this relation (Runcan

1 - Department of Health, Physical and Social Education, Lithuanian Sports University, Kaunas, Lithuania. 
and Iovu, 2013). It is not clear what potential mediating variables can account for the relationship between EI and psychological wellbeing. For instance, research by Zeidner et al. (2012) indicates some mediating variables in the EI-wellbeing relationship. A possible variable to mediate the association between EI and wellbeing/psychological wellbeing is social support (Kong et al., 2012). This finding has been also confirmed in athletic samples (Chen, 2013).

Researchers have identified that individuals who perceive much social support from others report greater wellbeing or wellbeing indicators such as life satisfaction (Gallagher and Vella-Brodrick, 2008). Gallagher and VellaBrodrick (2008) reported that perceived social support functioned as a partial mediator of the relationship between EI and wellbeing, as well as between EI and life satisfaction (Kong et al., 2012). Thus, we might expect that perceived social support would be a mediator in the association between EI and psychological wellbeing.

Another potential mediator of the relationship between EI and wellbeing is perceived stress. Perceived stress mediated the association between EI and wellbeing indicators, specifically life satisfaction and happiness (RuizAranda et al., 2014). Previous studies in nonathletic samples have found that EI is a skill that minimizes perceived stress and negative consequences of stress (Montes-Berges and Augusto, 2007). A considerable body of evidence from non-athletic samples suggests that EI is a mechanism that could predict perceived stress (Edward and Warelow, 2005; Slaski and Cartwright, 2002).

Several studies (Gallagher and VellaBrodrick, 2008; Montes-Berges and Augusto, 2007) have shown that the relationship between EI and perceived social support and its effect on wellbeing goes beyond main effects. The association between EI, perceived social support and wellbeing seems to be more complex, with EI and perceived social support being predictors of wellbeing. Thus, EI is likely to be associated with greater wellbeing by means of the greater perceived social support.

Additionally, perceived social support has been found to mediate the relationship between EI and other protective or vulnerability factors, for instance perceived stress (e.g., Montes-Berges and Augusto, 2007). Other authors have demonstrated that, beyond the direct influences of EI and perceived social support on wellbeing, emotional abilities and social support might have a significant influence on perceived stress in the prediction of well-being (Chao, 2012; Gohm et al., 2005). It can be hypothesized that perceived social support and perceived stress are sequential mediators in the association between EI and psychological wellbeing in a male athletic sample. Current Study

The aim of this study was to investigate mediating effects of perceived social support and perceived stress between EI and wellbeing over a 3-month period using structural equation modelling (SEM). This study is the first to test the full sequence of associations between EI, wellbeing, perceived social support and perceived stress in an athletic sample. Considering the studies of non-athletic samples that have reported relationships of EI and wellbeing (Gallagher and Vella-Brodrick, 2008), perceived social support (Gallagher and Vella-Brodrick, 2008; Kong et al., 2012), and perceived stress (Kong et al., 2012; Ruiz-Aranda et al., 2014), it was predicted in this study that perceived social support and perceived stress might play a mediating role in the EIpsychological wellbeing relationship in male athletes. The topicality of the present study is based on the idea that research investigating relationships of EI and wellbeing in non-athletic populations has failed to account for the specific characteristics of the athlete population.

A sample of male athletes was chosen because gender differences have been explored in three studies with athletic samples (Costarelli and Stamou, 2009; Dunn et al., 2007; Laborde et al., 2014); moreover, men outside athletic samples experience a lower incidence of depressive and anxiety disorders than women (Nolen-Hoeksema, 2000). Some empirical evidence regarding gender differences in wellbeing has been also reported (Malinauskas and Dumciene, 2017). For these reasons, it seemed necessary to analyze the role of EI in wellbeing for male athletes because the role of EI in the wellbeing of female samples (though only in non-athletic samples) has already been analyzed (Kong et al., 2012). Based on previous studies, two hypotheses were proposed:

Hypothesis 1: Perceived social support and perceived stress will be single mediators in 
the association between EI and wellbeing.

Hypothesis 2: Perceived social support and perceived stress will be sequential mediators in the association between EI and wellbeing.

\section{Methods}

\section{Study Design}

A 3-month prospective design was chosen for this study in order to investigate a mediation model in which perceived social support and perceived stress were conceptually viewed as a causal mechanism accounting for the EI/wellbeing link. EI is known to be associated with wellbeing (Schutte et al., 2002), as well as with perceived social support (Kong et al., 2012), but the psychological mechanism through which EI might predict wellbeing remains unclear (Ruiz-Aranda et al., 2014) in both non-athletic and athletic samples. Moreover, we examined the mediator role of perceived social support and perceived stress in the relationship between EI and wellbeing indicators.

\section{Participants}

Participants were drawn from a randomly selected sample of five universities in Lithuania. The sample of varsity male athletes was selected using simple random sampling. A total of 412 male varsity athletes participated in the survey. Fourteen students did not accurately complete the survey and were excluded from the analysis because of missing responses. The final sample thus consisted of 398 male varsity athletes. The mean age of the participants was $23.48(S D=2.67)$ years.

\section{Instruments}

\section{Schutte Self-Report Inventory (SSRI)}

Emotional intelligence was measured using the SSRI, which was validated by Schutte et al. (1998). The SSRI, otherwise known as the EIS (Emotional Intelligence Scale), the SEI (Self-Report Emotional Intelligence) and the Schutte Emotional Intelligence Scale (SEIS), assesses El based on selfreported responses. This instrument is beneficial for the way that it divides emotional intelligence into four separate components (Palmer, 2003), namely: ability to use own positive emotional experience (optimism), ability to assess and express emotions (appraisal), ability to understand and analyze emotions (social skills) and ability to manage emotions (utilization). It consists of 33 Likert items answered on a 5-point scale, ranging from 1 (strongly disagree) to 5 (strongly agree). By averaging items, we calculated a total indicator of emotional intelligence. The internal consistency for this research was good $(\alpha$ $=.86$ ). The Lithuanian version of the SSRI produced an internal consistency value of .79 and a test-retest reliability coefficient of .84 for the overall questionnaire (Malinauskas and Sniras, 2010).

Multidimensional Scale of Perceived Social Support (MSPSS)

Individual perceptions of social support were assessed using the Multidimensional Scale of Perceived Social Support (MSPSS; Zimet et al., 1988). The MSPSS was shown to be psychometrically sound, with good reliability, factor validity and adequate construct validity (Zimet et al., 1988). The authors specifically constructed the scale to be economical, thus providing for the use of the instrument in conjunction with other measures. The MSPSS consists of 12 items describing three different subscales: Family Support (4 items), Friend Support (4), and Significant Other Support (4). Respondents used a 7-point Likert-type scale (very strongly disagree to very strongly agree) to rate each item. The validity and reliability of the scale has been reported to be satisfactory (Kazarian and McCabe, 1991; Zimet et al., 1988). Cronbach's alpha was .71 for the entire scale, and $.69, .74$ and .76 for the subscales of perceived support from family, friends and significant other, respectively (Kazarian and McCabe, 1991; Zimet et al., 1988). The Lithuanian version of the MSPSS reported an internal consistency value of .61 for the entire scale (Malinauskas, 2010). A total indicator of perceived social support (MSPSS-Total) was calculated by averaging items in the present study. The coefficient alpha for the whole scale was .71 for the present sample.

Perceived Stress Scale-10 (PSS-10)

The Perceived Stress Scale-10 is a 10item, self-report inventory that assesses the degree to which situations in an individual's life are appraised as stressful (Cohen et al., 1983). PSS-10 items were designed to determine how much respondents feel their lives are unpredictable, uncontrollable, and overwhelming. These three issues have been repeatedly found to be central components of the experience of stress. Respondents indicated how 
often they felt or thought a certain way on a 5point Likert scale $(0=$ never to $4=$ very often $)$. One item reads, for example, "How often have you felt that you were unable to control the important things in your life?". The Lithuanian version of the PSS-10 showed an internal consistency value of 0.90 (Malinauskas, 2010). The coefficient alpha for the total scale was .88 for the present sample.

Ryff Psychological Wellbeing Scale (RPWBS)

The RPWBS was created by Ryff (1989)

and later revised by Ryff and Keyes (1995). This 54-item scale was used in this study. The RPWBS consists of a series of items reflecting six aspects of psychological wellbeing (PWB): autonomy, environmental mastery, personal growth, positive relations with others, purpose in life and selfacceptance (Ryff, 1989). Respondents are required to rate the items using a 6-point Likert scale ranging from 1 (strongly disagree) to 6 (strongly agree). The reported internal consistency of the subscales making up the 54-item scale ranges from .63 to .81 . Like Sheldon and Lyubomirsky (2006), we calculated a total psychological wellbeing score not by summing the scores for the six subscales, but by averaging. When items are summed, their variances are summed, but when items are averaged, their variance is reduced. Thus, averaging items decreases the noise in a set of scale scores. In this study the alpha coefficient for the total score was .73. The Lithuanian version of the RPWBS has reported internal consistency of .84 (Malinauskas and Dumciene, 2017).

\section{Procedures}

Participants were administered the Schutte Self-Report Inventory during scheduled seminar time. After 12 weeks, all participants completed the Multidimensional Scale of Perceived Social Support, Perceived Stress Scale, and Ryff Psychological Wellbeing Scale.

This research meets the ethical guidelines, including adherence to the legal requirements of the country where this study was conducted. Ethical clearance was obtained from the Committee for Social Sciences Research Ethics of the Lithuanian Sports University.

\section{Statistical Analysis}

To address the primary objectives, a series of structural equation models (SEMs) assessing associations among EI, perceived social support, perceived stress, and psychological wellbeing was estimated using MPlus, version 7.0 (Muthén and Muthén, 2012). A series of SEMs was performed to identify the best data fit to the model according to the model fit indices. These indices included the chi-square test of model fit with $p$-value, the comparative fit index (CFI), the Tucker-Lewis index (TLI), root mean square error of approximation (RMSEA) and standardized root mean square residual (SRMR). A CFI above .95, TLI values above .90 and RMSEA values below .08 indicate acceptable model fits (Browne and Cudeck, 1993; $\mathrm{Hu}$ and Bentler, 1998). The structural models were tested via the maximum likelihood estimation method. In order to find the best model, we additionally examined the Akaike Information Criterion (AIC: Akaike, 1987) to determine whether the hypothesized model was a better fit.

The significance of the mediating effects of perceived social support and perceived stress was tested using the bootstrap estimation procedure. In order to test the significance of indirect effects, we used one thousand 95\% bootstrap confidence intervals (CI). Bootstrapping is a recommended analytic technique that involves repeated random sampling observations with replacement from the dataset (Hayes, 2009). An indirect effect was considered to be significant at the .05 level if the 95\% CI from 1,000 bootstrap samples did not include zero. Only standardized significant estimates are presented in Figure 1.

\section{Results}

\section{Descriptive and Correlation Analyses}

Table 1 presents means, standard deviations, skewness and kurtosis, standard errors of skewness and kurtosis, and Cronbach reliability estimates for all variables. All variables were checked for skewness and kurtosis and were considered to have acceptable distributions, thus making it possible to use structural equation modelling.

Pearson correlation coefficients among students' EI and other variables were calculated and are presented in Table 2. As no correlation exceeded .70, the assumption of multicollinearity was not violated. As predicted, students' EI was positively correlated with perceived social support $(r=.31 ; p<.01)$ and wellbeing $(r=.57$; 
$p<.01)$, and negatively with perceived stress ( $r=-$ $.18 ; p<.01)$. Negative relations were identified between perceived stress and perceived social support $(r=-.24 ; p<.01)$ and wellbeing $(r=-.23 ; p$ $<$ 01). Effect size was tested using Cohen determination coefficient. According to Kotrlik and Williams (2003), the results confirmed the high correlation between EI and perceived stress, perceived social support, and wellbeing (Cohen's d ranged from .19 to 1.90).

Primary Analysis

Structural equation modeling with latent variables was used to examine the main hypotheses. We conducted analysis of the covariance matrices using MPlus, version 7.0 (Muthén and Muthén, 2012), and solutions were generated using maximum-likelihood estimation. EI was modeled as a latent factor with four indicators, namely optimism, appraisal, social skills, and utilization. Perceived social support was modeled as a latent factor with three indicators (family support, friend support, and significant other support), and psychological wellbeing was modeled as a latent factor with six indicators: optimism, appraisal, social skills, utilization, autonomy, environmental mastery, personal growth, positive relations with others, purpose in life and self-acceptance. Perceived stress as a latent variable was represented by two randomly computed parcels (Perceived stress1 and Perceived stress2; Williams and O'Boyle, 2008).

The quality of the measurement model (i.e., the relations between indicators and latent constructs) was assessed through CFA (confirmatory factor analysis). The measurement model involved four latent constructs (EI, perceived social support, perceived stress, and psychological wellbeing) and 15 observed variables. An initial test of the measurement model generated a good fit to the data: $\chi^{2}(84, N=$ 398 ) $=80.28, p=.59 ; \chi^{2} / \mathrm{df}=.96$ (less than the criteria of 3; Kline, 2011); RMSEA = .000; SRMR = .033; $\mathrm{CFI}=1.00$. All factor loadings for the indicators on the latent variables were significant (i.e., $\geq .50, p<.001$ ), demonstrating that all the latent constructs were well represented by their indicators. Moreover, as reported in Table 1, all the latent constructs were significantly correlated in conceptually expected ways $(p<.01)$. Having established an appropriately fitting measurement model, we estimated structural models testing the hypothesized relations between the latent variables.

Mediation Analysis

In order to identify the best model, we assessed four alternative models. We first tested a partially-mediated model (Model 1) with two simple mediators and a direct path from EI to psychological wellbeing, which revealed an excellent fit to the data $\left(\chi^{2}(85, N=398)=102.78, p\right.$ $=.09 ; \chi^{2} / \mathrm{df}=1.21$ (less than the criteria of 3 ); RMSEA = .023; SRMR = .033; CFI = .99; TLI = .99; AIC $=4910.20)$. Later on, a fully-mediated model (Model 2) was tested, which also showed a good fit to the data $\left(\chi^{2}(86, N=398)=143.03, p<.001\right.$; $\chi^{2} / \mathrm{df}=1.66$ (less than the criteria of 3 ); RMSEA = $.041 ; \mathrm{SRMR}=.033 ; \mathrm{CFI}=.96 ; \mathrm{TLI}=.95 ; \mathrm{AIC}=$ 4948.45).

In order to identify the best model, a path from perceived social support to perceived stress was added to the initial model, and the results showed an excellent fit to the data $\left(\chi^{2}(84\right.$, $N=398)=81.25, p=.56 ; \chi^{2} / \mathrm{df}=.97$ (less than the criteria of 3 ); RMSEA $=.000$; $\mathrm{SRMR}=.033$; $\mathrm{CFI}=$ 1.00; TLI $=1.00 ; \mathrm{AIC}=4890.67 ;$ Model 3 - partial mediated model with two sequential mediators).

Next, a full mediated model with two sequential mediators (Model 4) was tested, which showed a good fit to the data $\left(\chi^{2}(85, N=\right.$ $398)=122.12, p<.01 ; \chi^{2} / \mathrm{df}=1.44$ (less than the criteria of 3); RMSEA $=.023$; SRMR $=.033$; $\mathrm{CFI}=$ $.97 ; \mathrm{TLI}=.97 ; \mathrm{AIC}=4929.54$ ).

In respect to the better fit indices, they suggested that the fit of Model 1 and Model 3 were more satisfactory. When Model 1 and Model 3 were compared, the significant chi-square difference, $\Delta \chi 2(1, N=398)=21.53, p<0.001$, as well as the smaller AIC, indicated that the additional path from perceived social support to perceived stress significantly contributed to the model. Taking these factors into consideration, Model 3 was selected as the best model (Figure 1).

Standardized $b$ weights for direct effects in mediation analysis are presented in Figure 1. Structural paths were significant at the 01 level, with the exception of the path from perceived social support to wellbeing and of the path from EI to perceived stress, which were significant at the .05 level. About $49.3 \%$ of the variance in wellbeing was explained by EI, perceived social 
support and perceived stress in the mediation model. As reported in Table 3 and Figure 1, the bootstrap method revealed that perceived social support partially mediated the association between EI and wellbeing ( $\beta=.012, b=.042,95 \%$ CI .002-0.031 (does not include zero)).

Perceived stress also partially mediated the association between EI and wellbeing ( $\beta=.012$, $\mathrm{b}=.041,95 \%$ CI .004-0.027 (does not include zero)). These findings support Hypothesis 1. Therefore, perceived social support and perceived stress are sequential mediators in the relationship between EI and wellbeing ( $\beta=.006, b=.020,95 \%$ CI .0020.015 (does not include zero)). However, these findings do not support Hypothesis 2.

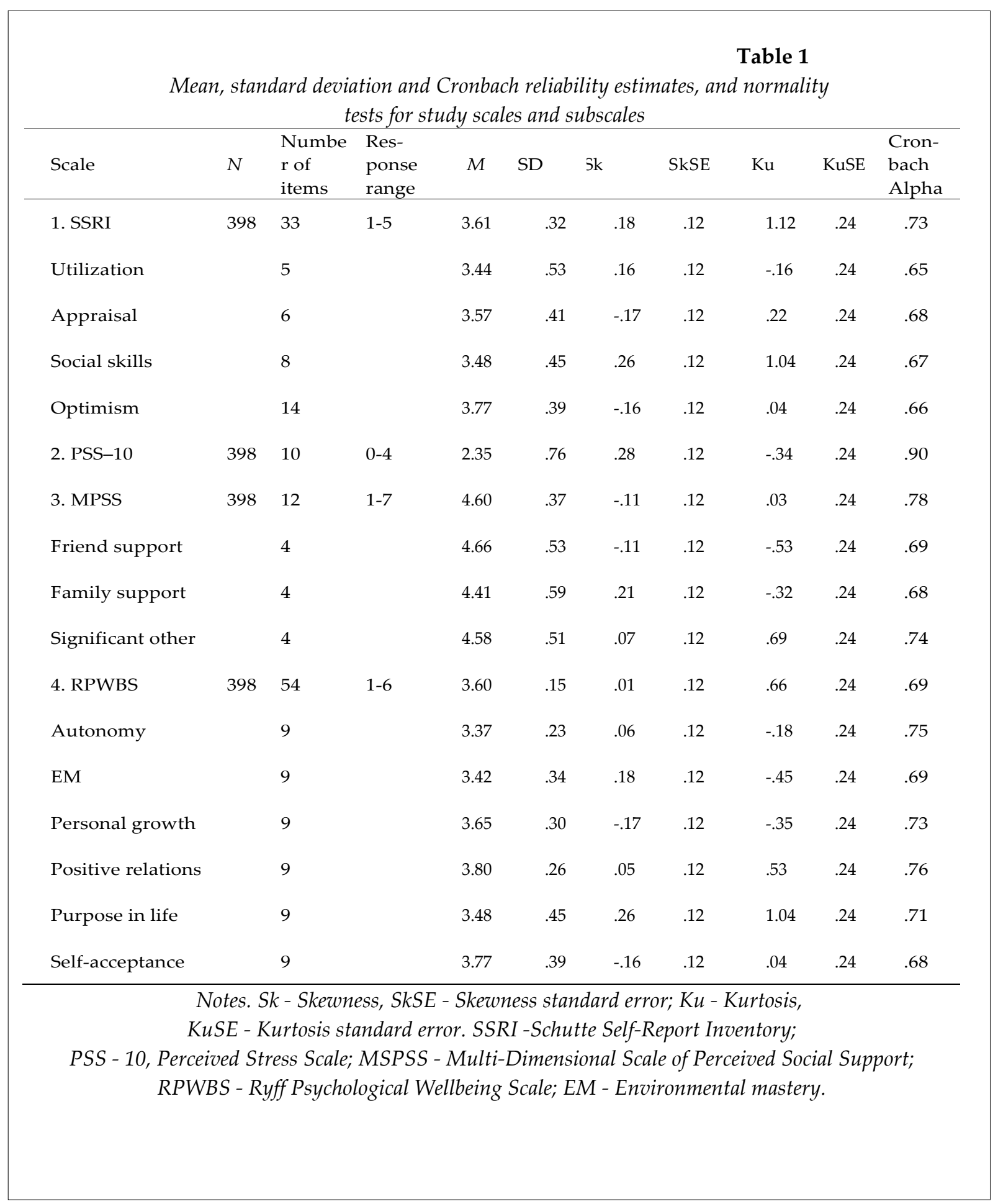


Table 2

2. Utilization $\quad-\quad .23^{* *} .42^{* *} .37^{* *}-.15^{* *} \quad .21^{* *} \quad .17^{* *} \quad .18^{* *} .10^{*} \quad .20^{*:} \quad .19^{*} .17^{* *} .10^{*} .11^{*} .16^{* *} \quad .22^{* *}$

3. Appraisal $.29^{* *} \cdot 35^{* *}-.17^{* *} \quad .14^{* *} \quad .15^{* *} \quad .10^{*} \quad .11^{*} \quad .17^{* *} \quad .15^{*} \cdot 11^{*} \quad .17^{* *} \cdot 18^{* *} \cdot 23^{* *} \quad .25^{* *}$

4. Social skills

$-\quad .45^{* *}-.16^{* *} .12^{*} \quad .14^{* *} \quad .14^{* *} .09 \quad .16^{*:} \quad .14^{*} .11^{*} \quad .10^{*} .17^{* *} .14^{* *} \quad .18^{* *}$

5. Optimism

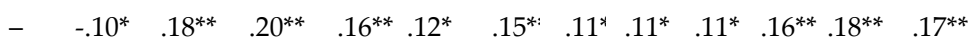

6. PSS-10

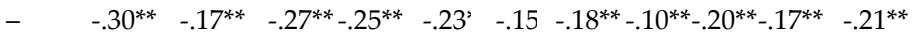

7. MPSS

$-\quad .63^{* *} \quad .66^{* *} .69^{* *} \quad .23^{*:} .15^{*} .11^{*} .17^{* *} \cdot 17^{* *} \cdot 21^{* *} .16^{* *}$

8. FrS

$-\quad .64^{* *} \cdot .56^{* *} \quad .15^{*} \quad .10^{*} \cdot 10^{*} \cdot 15^{* *} \cdot 13^{* *} \cdot 12^{*} \cdot 12^{*}$

9. FaS

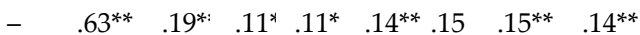

10. $\mathrm{SO}$

$-\quad .17^{*:} \quad .10^{*} .09 \quad .14^{* *} .12^{*} .15^{* *} .11^{*}$

11. RPWBS

$-\quad .51^{*} .49^{* *} \cdot 53^{* *} \cdot 50^{* *} \cdot 53^{* *} .52^{* *}$

12. Autonomy

- $.15^{* *} \cdot 14^{* *} \cdot 20^{* *} \cdot 11^{*} \quad .23^{* *}$

13. EM

$\begin{array}{llll}- & .09 & .12^{*} .11^{*} & .10^{*}\end{array}$

14. PG

$-.10^{*} .19^{* *} .11^{*}$

15. PR

$-.16^{* *} .13^{* *}$

16. PL

$-.32^{* *}$

17. SA

Notes. ${ }^{*} p<.05 ;{ }^{* *} p<.01$. SSRI-Schutte Self-Report Inventory;

PSS - 10, Perceived Stress Scale; Perceived stress1-Perceived stress $2=$ two parcels of perceived stress.

MSPSS - Multi-Dimensional Scale of Perceived Social Support;

RPWBS - Ryff Psychological Wellbeing Scale; EM - Environmental mastery;

FrS - Friend support; FaS - Family support; SO - Significant other; PG - Personal growth;

PR - Positive relations; PL - Purpose in life; SA-Self-acceptance. 


\begin{tabular}{|c|c|c|c|c|}
\hline \multicolumn{5}{|c|}{ Bootstrap estimates of the direct and indirect effects of EI on welbeing } \\
\hline \multirow[t]{2}{*}{ Model pathways } & \multicolumn{2}{|c|}{ Parameter estimate } & \multicolumn{2}{|c|}{ Bias-corrected CI (95\%) } \\
\hline & $\begin{array}{l}\text { Unstandardized } \\
\quad(S E)\end{array}$ & Standardized $(S E)$ & Lower & Upper \\
\hline Direct effect & $.148(.033)$ & $.514(.074)$ & .085 & $.224^{*}$ \\
\hline Indirect effect via PSS & $.012(.006)$ & $.042(.021)$ & .002 & $.031^{*}$ \\
\hline Indirect effect via PS & $.012(.006)$ & $.041(.018)$ & .004 & $.027^{*}$ \\
\hline $\begin{array}{l}\text { Indirect effect via PSS } \\
\text { and PS }\end{array}$ & $.006(.003)$ & $.020(.008)$ & .002 & $.015^{*}$ \\
\hline Sum of indirect via PSS & $.018(.008)$ & $.063(.023)$ & .007 & $.038^{*}$ \\
\hline Sum of indirect via PS & $.018(.007)$ & $.062(.020)$ & .008 & $.036^{*}$ \\
\hline $\begin{array}{l}\text { Sum of indirect via PSS } \\
\text { and PS }\end{array}$ & $.006(.003)$ & $.020(.008)$ & .002 & $.015^{*}$ \\
\hline $\begin{array}{r}\text { Notes. } N=398 . P \\
C I-C \\
* \text { This } 95^{\circ} \\
\text { the ind }\end{array}$ & $\begin{array}{l}\text { Perceived Social } \\
\text { ence interval; SE } \\
\text { fidence interval } \\
\text { relationship is si }\end{array}$ & $\begin{array}{l}\text { Support; PS - Per } \\
\text { - standard error. } \\
\text { excludes zero; the } \\
\text { gnificant at } p<.0\end{array}$ & $\begin{array}{l}\text { ceived } \\
\text { refore, } \\
5 .\end{array}$ & \\
\hline
\end{tabular}

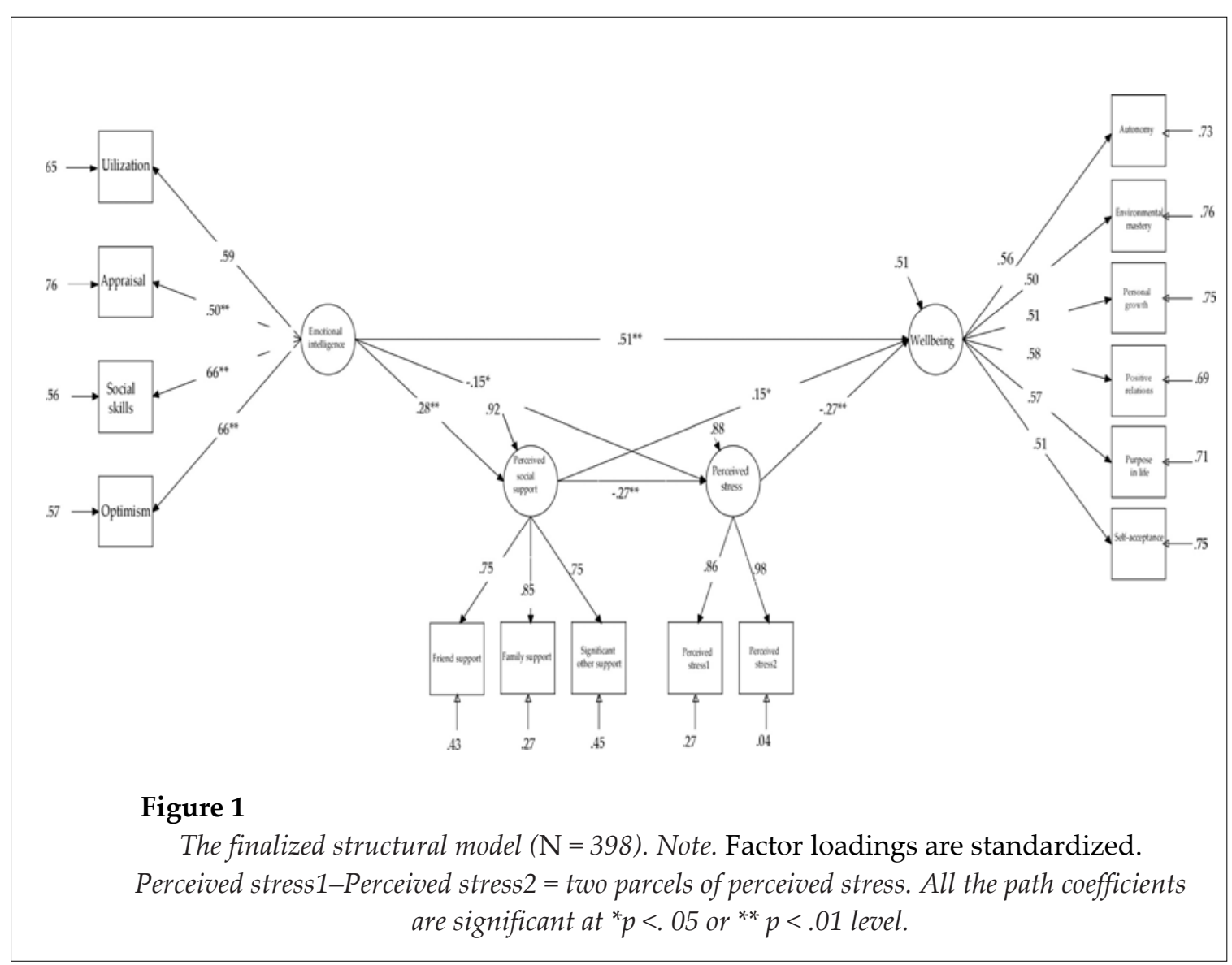




\section{Discussion}

In the present study, we made an attempt to extend previous cross-sectional works (Gallagher and Vella-Brodrick, 2008) by analysing the mediating role of perceived stress and perceived social support in the relationship between EI and psychological wellbeing after 3 months in a sample of varsity male athletes, much like Ruiz-Aranda et al. (2014) in their investigation with a sample of female students.

Hypothesis 1, which predicted that perceived social support and perceived stress would be single mediators in the association between EI and wellbeing, was confirmed. The main finding of the present study is that the path of EI $\rightarrow$ perceived social support $\rightarrow$ psychological wellbeing is significant. Another finding of the present study is the significant path of EI $\rightarrow$ perceived stress $\rightarrow$ psychological wellbeing. Consistent with previous studies in non-athletic samples, people with high trait EI are likely to perceive greater social support from others and report higher levels of wellbeing indicators (Schiffrin and Nelson, 2010). Our findings on the mediation of perceived social support in association between EI and wellbeing support the Gallagher and Vella-Brodrick's (2008) claim that perceived social support functioned as a partial mediator of the relationship between EI and wellbeing. The mediating effect of social support may be explained as individuals with higher EI being better able to recognize and manage emotions in others and have better opportunities to enhance their social support, which in turn contributes to an increase in their wellbeing. These results are in line with previous reports that social support functioned as a mediator of the relationship between EI and wellbeing indicators, specifically life satisfaction and happiness (Kong et al., 2012, 2015).

In the same way, the findings of the present study are similar to those by RuvalcabaRomero et al. (2017), who also confirmed the importance of both positive emotions and interpersonal relationships (like indicators of social support), which contributed with $50 \%$ of the variance to life satisfaction (like indicator of wellbeing) in the relationship between EI and life satisfaction. However, we did not find any investigations of athletic samples to compare with the findings of the present study.

The mediating effect of perceived stress may be explained by the fact that EI is a skill that minimizes perceived stress and the negative consequences of stress (Montes-Berges and Augusto, 2007) in non-athletic samples and that EI has been found to be a significant predictor of coping effectiveness as well as mental toughness in athletic samples (Cowden, 2016; Kaiseler et al., 2017).

Some authors have pointed out that EI and social support might significantly influence perceived stress in the prediction of wellbeing (e.g., Chao, 2012). We proposed that male varsity athletes with higher EI efficacy may have a propensity to experience greater support within the environment, such as from coaches and teammates, contributing to a decreased possibility of stress. We expected that perceived social support and perceived stress would be sequential mediators in the association between EI and wellbeing. Our results support Hypothesis 2. All pathways of EI $\rightarrow$ perceived social support $\rightarrow$ perceived stress $\rightarrow$ psychological wellbeing were significant, and the indirect effect of EI on wellbeing via perceived social support and perceived stress was statistically significant. That is, perceived social support and perceived stress mediated the association between EI and wellbeing. Our results are compatible with a study of a non-athletic sample (Perera and DiGiacomo, 2015), which found that social support and coping (like indicators of perceived stress) mediate the relationship between EI and psychological adjustment (like indicator of wellbeing).

Significant similar associations were found between perceived stress and diminished life satisfaction (like indicator of wellbeing) and among perceived stress and perceived social support interaction and diminished life satisfaction in the athletes with a major injury (Malinauskas, 2010). Some similar studies in the sport context have also confirmed that social support is an effective buffer between adverse sports events and negative responses (for instance, when competition was lost) and is associated with injured athletes' well-being (Bianco and Eklund, 2001; Lu and Hsu, 2013; Rees and Hardy, 2000). The study by Katagami and Tsuchiya (2016) highlighted that perceived social 
support positively correlated with positive athletes' self-schema and the receipt of social support may be a key factor in athletes' psychological well-being not only for sport performance outcomes.

Interestingly, previous studies have analyzed the mediating role of social support between EI and wellbeing using cross-sectional designs in non-athletic samples, which does not allow causal conclusions (Gallagher and VellaBrodrick, 2008). In this study, however, we employed the 3-month prospective design to assess the mediators of the association between EI and wellbeing. Another strength of this study is its relatively large athletic sample.

Several limitations of the present study should be noted. The first limitation was that our results were limited to male varsity athletes, so the findings may not be generalizable to the entire population of varsity athletes in Lithuania. An expanded sample might be employed in further investigations, and future research should include the female population and analyze the existence of possible differences. The second limitation of the present study was that we relied on selfreported measures, making it likely that social desirability may have influenced the responses. Further research should investigate other possible mediators in the association between EI and wellbeing. Other variables may mediate the effect of EI on wellbeing, for instance, psychological skills, which are considered important predictors in relations between EI and psychological wellbeing in an athletic sample (Kajbafnezhad et al., 2012).

\section{Conclusions}

Results from structural equation modelling procedures indicated that perceived social support and perceived stress partially mediated the association between EI and wellbeing. Furthermore, the sequential mediation effects of perceived social support-perceived stress on the relations between EI and wellbeing were confirmed.

The current findings provide a scientific rationale for recommending coaches to strengthen the role of social support of male athletes and teach the athletes with lower EI efficacy to overcome the negative perceptive disadvantages (perceived stress) when competition was lost. Therefore, athletes with higher EI efficacy have a propensity to experience greater support within the environment, such as from coaches and teammates, contributing to a decreased possibility of stress. Taken together, the results of this study should be considered in training and competitions processes and encourage supportive training and a positive competitive environment for athletes because greater social support contributes to an increase in male athletes' wellbeing.

\section{References}

Akaike H. Factor analysis and AIC. Psychometrika, 1987; 52: 317-332

Aghdasi S, Kiamanesh AR, Ebrahim, AN. Emotional Intelligence and organizational commitment: testing the mediatory role of occupational stress and job satisfaction. Procedia Soc Behav Sci, 2011; 29: 1965-1976

Bianco T, Eklund RC. Conceptual considerations for social support research in sport and exercise settings: The case of sport injury. J Sport Exerc Psychol, 2001; 23: 85-107

Browne MW, Cudeck R. Single sample cross-validation indices for covariance structures. Multivariate Behav Res, 1989; 24: 445-455

Chao RCL. Managing perceived stress among college students: The roles of social support and dysfunctional coping. J Coll Couns, 2012; 15: 5-21

Chen LH. Gratitude and adolescent athletes' well-being: The multiple mediating roles of perceived social support from coaches and teammates. Soc Indic Res, 2013; 114: 273-285

Ciarrochi J, Deane F, Anderson S. Emotional intelligence moderates the relationship between stress and mental health. Pers Individ Dif, 2002; 32: 197-209 
Cohen S, Kamarck T, Mermelstein R. A global measure of perceived stress. J Health Soc Behav, 1983; 24: 385396

Costarelli V, Stamou D. Emotional intelligence, body image and disordered eating attitudes in combat sport athletes. J Exerc Sci Fit, 2009; 7: 104-111

Cowden RG. Mental toughness, emotional intelligence, and coping effectiveness: an analysis of construct interrelatedness among high-performing adolescent male athletes. Percept Mot Skills, 2016; 123: 737753

Dunn EW, Brackett MA, Ashton-James C, Schneiderman E, Salovey P. On emotionally intelligent time travel: Individual differences in affective forecasting ability. Pers Soc Psychol Bull, 2007; 33: 85-93

Edward KL, Warelow P. Resilience: When coping is emotionally intelligent. Journal of the J Am Psychiatr Nurses Assoc, 2005; 11: 101-102

Gallagher EN, Vella-Brodrick DA. Social support and emotional intelligence as predictors of subjective wellbeing. Pers Individ Dif, 2008; 44: 1551-1561

Gohm CL, Corser GC, Dalsky DJ. Emotional intelligence under stress: useful, unnecessary, or irrelevant? Pers Individ Dif, 2005; 39: 1017-1028

Hayes AF. Beyond Baron and Kenny: Statistical mediation analysis in the new millennium. Commun Monogr, 2009; 76: 408-420

Hu LT, Bentler P. Fit indices in covariance structure modeling: Sensitivity to underparameterized model misspecification. Psychol Methods, 1998; 3: 424-453

Kaiseler M, Poolton JM, Backhouse SH, Stanger N. The relationship between mindfulness and life stress in student-athletes: The mediating role of coping effectiveness and decision rumination. Sport Psychol, 2017; 31: 288-298

Kajbafnezhad H, Ahadi H, Heidarie A, Askari P, Enayati M. Predicting athletic success motivation using mental skills and emotional intelligence and its components in male athletes. J Sports Med Phys Fitness, 2012; 52: 551-557

Katagami E, Tsuchiya H. Effects of Social Support on Athletes' Psychological Well-Being: The Correlations among Received Support, Perceived Support, and Personality. Psychol, 2016; 7: 1741-1752

Kline RB. Principles and practice of structural equation modeling. New York: Guilford Press; 2011

Kong F, Zhao J, You X. Social support mediates the influence of emotional intelligence on mental distress and life satisfaction in Chinese young adults. Pers Individ Dif, 53: 513-517

Kong F, Ding K, Zhao J. The relationships among gratitude, self-esteem, social support and life satisfaction among undergraduate students. J Happiness Stud, 2015; 16: 477-489

Kotrlik JW, Williams HA. The incorporation of effect size in information technology, learning, and performance research. Inf Technol Learn Perform J, 2003; 121: 1-8

Laborde S, Dosseville F, Guillén F, Chávez E. Validity of the trait emotional intelligence questionnaire in sports and its links with performance satisfaction. Psychol Sport Exerc, 2014; 15: 481-490

Laborde S, Dosseville F, Allen MS. Emotional intelligence in sport and exercise: A systematic review. Scand J Med Sci Sports,2016; 26: 862-874

Lu FJ, Hsu Y. Injured athletes' rehabilitation beliefs and subjective well-being: The contribution of hope and social support. J Athl Train, 2013; 48: 92-98

Malinauskas R. The associations among social support, stress, and life satisfaction as perceived by injured college athletes. Soc Behav Pers, 2010; 38: 741-752

Malinauskas R, Dumciene A. Psychological wellbeing and self-esteem in students across the transition between secondary school and university: A longitudinal study. Psihologija, 2017; 50: 21-36 
Malinauskas R, Sniras S. Emotional intelligence among future sport pedagogues: Results of an empirical study. Sci Process Educ, 2010; 4: 81-88

Montes-Berges B, Augusto JM. Exploring the relationship between perceived emotional intelligence, coping, social support and mental health in nursing students. J Psychiatr Ment Health Nurs, 2007; 14: 163-171

Muthén LK, Muthén BO. Mplus user's guide. (7th edn). Los Angeles, CA: Muthén and Muthén; 2012

Nolen-Hoeksema S. The role of rumination in depressive disorders and mixed anxiety/ depressive symptoms. J Abnorm Psychol, 2000; 109: 304-311

Palmer BR. An analysis of the relationships between various models and measures of emotional intelligence. Swinburne: Swinburne University of Technology, School of Biophysical Sciences and Electrical Engineering, Centre for Neuropsychology; 2003

Perera HN, DiGiacomo M. The role of trait emotional intelligence in academic performance during the university transition: An integrative model of mediation via social support, coping, and adjustment. Pers Individ Dif, 2015; 83: 208-213

Petrides KV, Pita R, Kokkinaki F. The location of trait emotional intelligence in personality factor space. $\mathrm{Br} \mathrm{J}$ Psychol, 2007; 98: 273-289

Raedeke TD, Smith AL. Coping resources and athlete burnout: An examination of stress mediated and moderation hypotheses. J Sport Exerc Psychol, 2004; 26: 525-541

Rees T, Hardy L. An investigation of the social support experiences of high-level sports performers. Sport Psychol, 2000; 14: 327-347

Roohafza H, Feizi A, Afshar H, Mazaheri M, Behnamfar O, Hassanzadeh-Keshteli A, Adibi P. Path analysis of relationship among personality, perceived stress, coping, social support, and psychological outcomes. World J Psychiatry, 2016; 6: 248

Ruiz-Aranda D, Extremera N, Pineda-Galán C. Emotional intelligence, life satisfaction and subjective happiness in female student health professionals: the mediating effect of perceived stress. J Psychiatr Ment Health Nurs, 2014; 21: 106-113

Runcan PL, Iovu MB. Emotional intelligence and life satisfaction in Romanian university students: The mediating role of self-esteem and social support. Revista de Cercetare si Interventie Sociala, 2013; 40: 137148

Ruvalcaba-Romero NA, Fernández-Berrocal P, Salazar-Estrada JG, Gallegos-Guajardo J. Positive emotions, self-esteem, interpersonal relationships and social support as mediators between emotional intelligence and life satisfaction. J Behav Health Soc Issues, 2017; 9: 1-6

Ryff CD. Happiness is everything, or is it? Explorations on the meaning of psychological wellbeing. J Pers Soc Psychol, 1989; 57: 1069-1081

Ryff CD, Keyes CLM. The structure of psychological wellbeing revisited. J Pers Soc Psychol, 1995; 69: 719-727

Schiffrin HH, Nelson SK. Stressed and happy? Investigation of the relationship between happiness and perceived stress. J Happiness Stud, 2010; 11: 33-39

Schutte NS, Malouff JM, Hall LE, Haggerty DJ, Cooper JT, Golden CJ, Dornheim L. Development and validation of a measure of emotional intelligence. Pers Individ Dif, 1998; 25: 167-177

Schutte NS, Malouff JM, Simunek M et al. Characteristic emotional intelligence and emotional well-being. Cogn Emot, 2002; 16: 769-785

Sheldon KM, Lyubomirsky S. Achieving sustainable gains in happiness: Change your actions, not your circumstances. J Happiness Stud, 2006; 7: 55-86

Slaski M, Cartwright S. Emotional intelligence training and its implications for stress, health and performance. Stress Health, 2003; 19: 233-239 
Smith RE, Smoll FL, Barnett NP. Reduction of children's sport performance anxiety through social support and stress-reduction training for coaches. J Appl Dev Psychol, 1995; 16: 125-142

Williams LJ, O'Boyle Jr EH. Measurement models for linking latent variables and indicators: A review of human resource management research using parcels. Hum Resour Manage Rev, 2008; 18: 233-242

Zeidner M, Matthews G, Roberts RD. The emotional intelligence, health, and well-being nexus: What have we learned and what have we missed? Appl Psychol Health Well, 2012; 4: 1-30

Zimet GD, Dahlem NW, Zimet SG, Farley GK. The multidimensional scale of perceived social support. J Pers Assess, 1988; 52: 30-41

\section{Corresponding author:}

\section{Professor, Dr. Romualdas Malinauskas}

Department of Health, Physical and Social Education, Lithuanian Sports University, Sporto 6, 44221 Kaunas, Lithuania

Phone No: 0037037302672

Fax: 0037037204515

E-mail: romas.malinauskas@lsu.lt 